\title{
CHARACTERIZATION OF THE SMOOTHNESS OF FUNCTIONS WITH A GIVEN ORDER OF APPROXIMATION BY POLYNOMIALS ON THE REAL LINE
}

\author{
V. M. FEDOROV \\ Department of Mathematics and Mechanics, Moscow State University \\ Moscow, U.S.S.R.
}

We study the structural properties of functions with a prescribed order of best approximation by algebraic polynomials in weighted spaces.

It is well known that the order of best uniform approximation by polynomials on the interval $[-1,1]$ for a function $f \in \operatorname{Lip}_{a}[-1,1]$, i.e.

$$
|f(x)-f(y)| \leqslant M|x-y|^{\alpha}, \quad x, y \in[-1,1],
$$

is equal to $1 / n^{\alpha}$; however, conversely one can only show that functions with that order of best uniform approximation are in $\operatorname{Lip}_{\alpha / 2}[-1,1]$. This means that in this case there is a gap between the direct and inverse theorems. From the results of Nikol'skii [11], Timan [14] and Dzyadyk [2] it follows that in order to obtain a constructive characterization of the class $\operatorname{Lip}_{a}[-1,1]$ one has to consider the order of approximation at each individual point of $[-1,1]$. Analogously, in order to obtain a structural characterization of the class of functions with a given order of best uniform approximation on $[-1,1]$, one has to take into account the smoothness of the function at each point of the interval. For the $L^{p}[-1,1]$ norm, this idea was investigated in M. K. Potapov's papers [12], [13].

Denote by $L^{p}(I, \varrho)$ the space of all Lebesgue measurable functions $f$ such that the product $f \varrho$ is integrable with exponent $p$ on the interval $I$ of the real line. This is a Banach space with the norm

$$
\|f\|_{L^{p}(I, Q)}=\left(\int|f(x) \varrho(x)|^{p} d x\right)^{1 / p}, \quad p \geqslant 1 .
$$


We also introduce the following notation:

$$
\begin{gathered}
\varrho(x)=\exp \left(-|x|^{\gamma}\right), \\
X^{p}=L^{p}((-\infty, \infty), \varrho), \quad X^{\infty}=L^{\infty}((-\infty, \infty), \varrho), \\
E_{n}(f)_{X^{p}}=\inf _{c_{k}}\left\|f(x)-\sum_{k=0}^{n-1} c_{k} x^{k}\right\|_{X^{p}} .
\end{gathered}
$$

Our main result is the following.

Theorem A. A function $f \in X^{p}$ satisfies

$$
E_{n}(f)_{X^{p}}=O\left(1 / n^{\alpha}\right), \quad n \rightarrow \infty,
$$

for a given $\alpha>0$ if and only if

$$
\left\|\Delta_{h}^{r}(f, x)\right\|_{x} p_{h}=O\left(h^{\beta}\right), \quad h \rightarrow 0_{+},
$$

where $\Delta_{h}^{r}(f, x)$ is the $r$-th difference of $f$ with step $h>0, \gamma \geqslant 2, \beta=\alpha \gamma /(\gamma-1)$, $X_{h}^{p}=L^{p}\left(I_{h}, \varrho\right)$, and

$$
I_{h}=\left[-(1 / h)^{1 /(\gamma-1)},(1 / h)^{1 /(\gamma-1)}\right] .
$$

The method used below for proving Theorem A admits further generalizations to a class of weight functions $\varrho$ satisfying certain conditions. In particular, if $\varrho$ satisfies Dzhrbashchyan's conditions [1], then the assertions of Theorem 1 and 2 below remain valid. On the other hand, if $\varrho$ satisfies Freud's conditions [5], then Theorems 1, 2, 3, as well as Theorem A, hold. The proofs are similar to those given below for the weights $\varrho(x)$ $=\exp \left(-|x|^{\gamma}\right), \gamma \geqslant 2$.

Note that Dzhrbashchyan's conditions are weaker than those of Freud and therefore embrace a larger class of weights. The function $\varrho(x)$ $=\exp \left(-|x|^{\gamma}\right), 1<\gamma<2$, does not satisfy Freud's conditions, however, Theorems 1 and 2 hold for it; as for Theorem 3, the question remains open. A more general and evidently more difficult problem arises: for what weight functions $\varrho$ do the direct and inverse theorems of approximation theory fit exactly each other? As shown in [8] and [3], in proving the direct and inverse theorems for the weight $\varrho(x)=\exp (-|x|)$ one has to take into account the order of approximation and order of smoothness at each point of the real line, which complicates the solution of the above problem considerably. Let us also note that in [4] this problem was solved for the $L^{p}$ norm on the real line and for the sup norm on the half-line for the weights $\varrho(x)=|x|^{\alpha} \exp \left(-|x|^{\gamma}\right), \gamma \geqslant 2$, with a singularity at zero.

\section{II}

In this section we prove a number of auxiliary statements which improve somewhat the well-known inequalities proved by Freud. 
LEMMA 1. Let $a_{n}=c n^{1 / \eta}, c>0$, and let $\lambda_{n}$ be the Christoffel function of the weight $\varrho$, i.e.

$$
\lambda_{n}(\xi)=\min _{P_{n}(\xi)=1} \int_{-a_{n}}^{a_{n}} P_{n}^{2}(x) \varrho(x) d x,
$$

where the minimum is taken over all algebraic polynomials $P_{n}$ of degree at most $n$ with $P_{n}(\xi)=1$. Then there are $c_{1}, c_{2}>0$ depending on $c$ and $\gamma$ only such that

$$
\lambda_{n}(\xi) \leqslant c_{2}(1 / n)^{(\gamma-1 ! / \gamma} \varrho(\xi)
$$

for $\xi \in\left[-a_{n}^{1}, a_{n}^{1}\right]$, where $a_{n}^{1}=c_{1} n^{1 / \gamma}$.

Proof. Let $x^{\prime}, \xi^{\prime} \in[-1,1]$ and $F_{n}\left(x^{\prime}\right)=T_{n}\left(x^{\prime}\right) T_{n-1}\left(\xi^{\prime}\right)-T_{n-1}\left(x^{\prime}\right) T_{n}\left(\xi^{\prime}\right)$, where $T_{n}\left(x^{\prime}\right)=\cos n \arccos x^{\prime}$ is the Chebyshev polynomial. Put

$$
Q_{n}\left(x^{\prime}\right)=\frac{F_{n}\left(x^{\prime}\right)}{\left(x^{\prime}-\xi^{\prime}\right) F_{n}^{(1)}\left(\xi^{\prime}\right)} .
$$

It is not difficult to check that the polynomials $Q_{n}$ satisfy the inequality (see [7])

$$
\int_{-1}^{1} Q_{n}^{2}\left(x^{\prime}\right) d x^{\prime} \leqslant c_{3} / n, \quad \xi^{\prime} \in[-1,1]
$$

where $c_{3}>0$ is an absolute constant.

Let $x, \xi \in\left[-a_{n}, a_{n}\right]$ and $x=a_{n} x^{\prime}, \xi=a_{n} \xi^{\prime}, S_{n}(x)=Q_{n}\left(x^{\prime}\right)$. Then

$$
S_{n}(\xi)=1, \quad \int_{-a_{n}}^{a_{n}} S_{n}^{2}(x) d x \leqslant c_{2}(1 / n)^{(\gamma-1) / \gamma}
$$

Choose $c_{1}>0$ so that

$$
r(x)=\gamma \operatorname{sign}(\xi)|\xi|^{\gamma-1}(x-\xi) \geqslant-n
$$

for $x \in\left[-a_{n}, a_{n}\right]$ and $\xi \in\left[-a_{n}^{1}, a_{n}^{1}\right]$. The function

$$
R_{m}(x)=\exp \left(|\xi|^{\gamma / 2}\right)\left(1+\frac{1}{2 m} r(x)\right)^{m}, \quad m=[n / 2]+1,
$$

is an algebraic polynomial of degree $m$ with

$$
R_{m}^{2}(\xi)=\exp \left(|\xi|^{\gamma}\right), \quad R_{m}^{2}(x) \leqslant \exp \left(|x|^{\gamma}\right)
$$

for $x \in\left[-a_{n}, a_{n}\right]$ and $\xi \in\left[-a_{n}^{1}, a_{n}^{1}\right]$. Therefore if

$$
P_{n+1}(x)=S_{m}(x) R_{m}(x) R_{m}^{-1}(\xi)
$$


then

$$
\lambda_{n+1}(\xi) \leqslant \int_{-a_{n}}^{a_{n}} P_{n+1}(x) \varrho(x) d x \leqslant \varrho(\xi) \int_{-a_{n}}^{a_{n}} S_{m}^{2}(x) d x \leqslant c_{4}(1 / n)^{(\gamma-1) / \gamma} \varrho(\xi),
$$

which completes the proof of Lemma 1.

LEMMA 2. Let the function $\Gamma_{\xi}$ be defined by $\Gamma_{\xi}(x)=1$ for $x>\xi, \Gamma_{\xi}(x)=0$ for $x \leqslant \xi$. Then there is $c_{1}>0$ depending on $c$ and $\gamma$ only such that

$$
E_{n}\left(\Gamma_{\xi}\right)_{X_{n}^{1}} \leqslant c_{1}(1 / n)^{(\gamma-1) / \gamma} \varrho(\xi)
$$

where $X_{n}^{1}=L^{1}\left(I_{n}, \varrho\right), I_{n}=\left[-a_{n}, a_{n}\right]$.

Proof. Let $n=2 m-1$. Then there are points $\xi_{v} \in I_{n}, v=1, \ldots, m$, including a given point $\xi=\xi_{\mu}$, such that the following quadrature formula holds for all algebraic polynomials of degree at most $n$ [9]:

$$
\int_{I_{n}} P_{n}(x) \varrho(x) d x=\sum_{v=1}^{m} \lambda_{n}^{v} P_{n}\left(\xi_{v}\right)
$$

Using Markov's interpolation lemma [9], we find algebraic polynomials $\boldsymbol{P}_{n}$ and $Q_{n}$ of degree $n-1$ such that

$$
\begin{gathered}
P_{n}\left(\xi_{v}\right)= \begin{cases}1, & v=1, \ldots, \mu-1, \\
0, & v=\mu, \ldots, m,\end{cases} \\
Q_{n}\left(\xi_{v}\right)= \begin{cases}1, & v=1, \ldots, \mu, \\
0, & v=\mu+1, \ldots, m,\end{cases} \\
P_{n}(x) \leqslant \Gamma_{\xi}(x) \leqslant Q_{n}(x), \quad x \in I_{n} .
\end{gathered}
$$

Computing the integral by the above quadrature formula we obtain

$$
\begin{aligned}
E_{n}\left(\Gamma_{\xi}\right)_{X_{n}^{1}} & \leqslant \int_{I_{n}}\left[Q_{n}(x)-P_{n}(x)\right] \varrho(x) d x=\lambda_{m}^{\mu} \\
& \leqslant \lambda_{m}(\xi) \leqslant c_{2}(1 / n)^{(\nu-1) / \gamma} \varrho(\xi),
\end{aligned}
$$

where $c_{2}$ comes from Lemma 1 and $\xi \in\left[-a_{n}^{1}, a_{n}^{1}\right], a_{n}^{1}=c_{1} n^{1 / \gamma}$. The same estimate is valid for $\xi \in\left[-a_{n}, a_{n}\right] \backslash\left[-a_{n}^{1}, a_{n}^{1}\right]$, since then

$$
E_{n}\left(\Gamma_{\xi}\right)_{X_{n}^{1}} \leqslant \int_{|\xi|}^{\infty} \varrho(x) d x \leqslant \gamma^{-1}|\xi|^{\gamma-1} \varrho(\xi)
$$

where the last inequality is easily verified by integration by parts. The proof of Lemma 2 is complete.

LEMMA 3. Suppose $f$ is a function locally integrable with exponent $p$, $1 \leqslant p<\infty$; if $p=\infty$ we assume $f$ to be locally bounded. Denote by $v_{m}(f, x)$, $m=[(n-1) / 2]$, the de la Vallée Poussin means of the Fourier series of $f$ with 
respect to the orthonormal system of the algebraic polynomials $P_{v}$ with the weight $\varrho^{2}$ on $I_{n}=\left[-a_{n}, a_{n}\right]$. Then there is $c_{1}>0$ depending on $c$ and $\gamma$ only such that

$$
\left\|v_{m}(f)\right\|_{X_{n}^{p}} \leqslant c_{1}\|f\|_{X_{n}^{p}}, \quad X_{n}^{p}=L^{p}\left(I_{n}, \varrho\right) .
$$

Proof. We first consider the case $p=\infty$. From the recurrence formula for the orthonormal system $P_{v}$ :

$$
y P_{v}(y)=\frac{d_{v}}{d_{v+1}} P_{v+1}(y)+b_{v} P_{v}(y)+\frac{d_{v-1}}{d_{v}} P_{v-1}(y),
$$

where $d_{v}>0$ is the leading coefficient of $P_{v}$, we obtain

$$
d_{v} / d_{v+1} \leqslant\left\{\int_{i_{n}}\left[y P_{v}(y) \varrho(y)\right]^{2} d y\right\}^{1 / 2} \leqslant c n^{1 / \gamma} .
$$

Denote by $V_{m}(x, y)$ the kernel of the de la Vallee Poussin means:

$$
V_{m}(x, y)=m^{-1} \sum_{k=m}^{2 m-1} \sum_{v=0}^{k} P_{v}(x) P_{v}(y)
$$

Applying the Christoffel-Darboux formula and the above inequality, we find that

$$
\begin{aligned}
\left\|(x-\cdot) V_{m}(x, \cdot)\right\|_{x_{n}^{2}} \leqslant m^{-1}\left\|\sum_{k=m}^{2 m-1} \frac{d_{k}}{d_{k+1}} P_{k+1}(x) P_{k}(\cdot)\right\|_{x_{n}^{2}} \\
+m^{-1}\left\|_{k=m}^{2 m-1} \frac{d_{k}}{d_{k+1}} P_{k}(x) P_{k+1}(\cdot)\right\|_{X_{n}^{2}} \\
\leqslant c_{2}(1 / n)^{(\gamma-1) / \gamma} \lambda_{2 m}^{-1 / 2}(x) .
\end{aligned}
$$

Moreover, by the Parseval identity,

$$
\left\|V_{m}(x, \cdot)\right\|_{X_{n}^{2}} \leqslant \lambda_{2 m}^{-1 / 2}(x)
$$

Hence the Schwarz inequality yields

$$
\begin{aligned}
\left|v_{m}(f, x)\right| & =\left|\int_{I_{n}} V_{m}(x, y) f(y) \varrho^{2}(y) d y\right| \\
& \leqslant\left\|\left(|x-y|+n^{1 / \gamma-1}\right) V_{m}(x, \cdot)\right\|_{x_{n}^{2}}\left\|\frac{f(\cdot)}{|x-\cdot|+n^{1 / \gamma-1}}\right\|_{x_{n}^{2}} \\
& \leqslant c_{3} n^{(1 / \gamma-1) / 2} \lambda_{2 m}^{-1 / 2}(x)\|f\|_{x_{n}^{\infty}} .
\end{aligned}
$$

As shown by Freud [6], the estimate from below of the Christoffel function has the same order as the estimate from above given in Lemma 1, the only difference being that the former holds for all $\xi \in I_{n}$. Thus Lemma 3 is proved for $p=\infty$. 
If $p=1$, by the extremal property of the norm we obtain

$$
\begin{aligned}
\left\|v_{m}(f, x)\right\|_{X_{n}^{1}} & =\sup _{\|g\|_{X_{n}^{\infty}} \leqslant 1} \int_{i_{n}} v_{m}(f, x) g(x) \varrho^{2}(x) d x \\
& =\sup _{\|g\|_{X_{n}^{\infty}} \leqslant 1} \int_{i_{n}} f(x) v_{m}(g, \dot{x}) \varrho^{2}(x) d x \leqslant c_{1}\|f\|_{X_{n}^{1}} .
\end{aligned}
$$

Thus Lemma 3 is valid for $p=\infty$ and $p=1$. In the case $1<p<\propto$ it suffices to apply the Riesz-Thorin interpolation theorem, which completes the proof.

\section{III}

In this section we prove three theorems which imply Theorem A.

THEOREM 1. Suppose that a locally absolutely continuous function $f$ has a derivative which is locally integrable with exponent $p$ if $1 \leqslant p<\infty$, and locally bounded if $p=\infty$. Then there is $c_{1}>0$ depending on $c$ and $\gamma$ only such that

$$
E_{n}(f)_{X_{n}^{p}} \leqslant c_{1}(1 / n)^{(\gamma-1) / \gamma}\left\|f^{(1)}\right\|_{X_{n}^{p}} .
$$

Proof. We first consider the case $p=1$. Suppose $f$ has a locally bounded variation; this is true in particular for functions satisfying the assumption of the theorem. By Nikol'skii's duality theorem,

$$
E_{n}(f)_{X_{n}^{1}}=\sup _{g \in Y_{n}^{\infty}} \int_{i_{n}} f(x) g(x) \varrho^{2}(x) d x,
$$

where $Y_{n}^{\infty}$ is the set of all measurable functions $g$ orthogonal to all algebraic polynomials $P_{n-1}$ of degree at most $n-1$ with $\|g\|_{x_{n}^{\infty}} \leqslant 1$. Put

$$
G(x)=\int_{i_{n}} \Gamma_{x}(t) g(t) \varrho^{2}(t) d t,
$$

where the function $\Gamma_{x}$ is defined in Lemma 2. Then it follows from Lemma 2 that for all $x \in I_{n}$

$$
\begin{aligned}
|G(x)| & =\left|\int_{I_{n}}\left[\Gamma_{x}(t)-P_{n-1}(t)\right] g(t) \varrho^{2}(t) d t\right| \\
& \leqslant E_{n}\left(\Gamma_{x}\right)_{X_{n}^{1}} \leqslant c_{2}(1 / n)^{(\gamma-1) / \gamma} \varrho(x) .
\end{aligned}
$$

Consequently, integration by parts gives

$$
\begin{aligned}
E_{n}(f)_{X_{n}^{1}} & =\sup _{g \in Y_{n}^{\infty}}\left\{-\int_{i_{n}} f(x) d G(x)\right\}=\sup _{g \in Y_{n}^{\infty}}\left\{\int_{i_{n}} G(x) d f(x)\right\} \\
& \leqslant c_{2}(1 / n)^{(\gamma-1) / \gamma} \int_{i_{n}} \varrho(x)|d f(x)| .
\end{aligned}
$$


Let now $p=\infty, m=[(n-1) / 2]$. Put

$$
P_{n-1}(x)=\int_{0}^{x} v_{m}\left(f^{(1)}, t\right) d t+f(0)
$$

Then $P_{n-1}$ is an algebraic polynomial of degree at most $n-1$ and

$$
f(x)-P_{n-1}(x)=\int_{i_{n}}\left[G_{x}(t)-Q_{m}(t)\right]\left[f^{(1)}(t)-v_{m}\left(f^{(1)} ; t\right)\right] \varrho^{2}(t) d t,
$$

where $Q_{m}$ is an arbitrary polynomial of degree at most $m$ and $G_{x}(t)=g^{-2}(t)$ for $t \in[0, x], G_{x}(t)=0$ for $t \notin[0, x]$. Hence using the above-proved assertion for $p=1$ we find that

$$
\begin{aligned}
E_{m+1}\left(G_{x}\right) x_{n}^{1} & \leqslant c_{3}(1 / n)^{(\gamma-1) / \gamma} \int_{i_{n}} \varrho(t)\left|d G_{x}(t)\right| \\
& \leqslant c_{3}(1 / n)^{(\gamma-1) / \gamma}\left(1+\varrho^{-1}(x)+2 \varrho^{-1}(x)-2\right) \\
& \leqslant c_{3} \cdot 3(1 / n)^{(\gamma-1) / \gamma} \varrho^{-1}(x) .
\end{aligned}
$$

Consequently, Lemma 3 and the above representation of the difference $f(x)$ $-P_{n-1}(x)$ show that

$$
\left\|f(x)-P_{n-1}(x)\right\|_{X_{n}^{\infty}} \leqslant c_{4}(1 / n)^{(\gamma-1) / \gamma}\left\|f^{(1)}\right\|_{X_{n}^{\infty}} .
$$

Thus the assertion of Theorem 1 is valid for $p=1$ and $p=\infty$.

Let $1 \leqslant p \leqslant \infty$. Then Lemma 3 yields the well-known property of the de la Vallée Poussin means:

$$
\left\|f-v_{m}(f)\right\|_{x_{n}^{p}} \leqslant c_{5} E_{m}(f)_{X_{n}^{p}}
$$

By the above, for $p=1$ or $p=\infty$ this yields

$$
\left\|f-v_{m}(f)\right\|_{X_{n}^{p}} \leqslant c_{1}(1 / n)^{(\gamma-1) / \gamma}\left\|f^{(1)}\right\|_{X_{n}^{p}}
$$

Applying now the Riesz-Thorin theorem to the operator

$$
A(F, x)=\left[f(x)-v_{m}(f, x)\right] \varrho(x), \quad F(x)=f^{(1)}(x) \varrho(x),
$$

we obtain for $1 \leqslant p \leqslant \infty$

$$
E_{2 m}(f)_{X_{n}^{p}} \leqslant c_{1}(1 / n)^{(\gamma-1) / \gamma}\left\|f^{(1)}\right\|_{X_{n}^{p}}
$$

Since $2 m<n$, Theorem 1 is proved.

Note that under the assumptions of Theorem 1 we have

$$
E_{n}(f)_{X^{p}} \leqslant c_{1}(1 / n)^{(\gamma-1) / \gamma}\left\|f^{(1)}\right\|_{X^{p}}
$$

provided $f^{(1)} \in X^{p}$. In the proof of Theorem 2 we will indicate a method of extending the estimates of best approximations from the intervals $I_{n}$ to the whole real line. 
For every function $f$ locally integrable with exponent $p$ if $1 \leqslant p<\infty$, and locally bounded if $p=\infty$, we define the modulus of continuity by

where

$$
\omega_{r}(f, \delta)_{X}{ }^{\prime}=\sup _{0<h \leqslant \delta}\left\|\Delta_{h}^{r}(f, x)\right\|_{X p^{p}},
$$

$$
\begin{gathered}
X_{h}^{p}=L^{p}\left(I_{h}, \varrho\right), \quad I_{h}=\left[-(1 / h)^{1 /(\gamma-1)},(1 / h)^{1 /(\gamma-1)}\right], \\
\Delta_{h}^{r}(f, x)=\sum_{v=0}^{r}(-1)^{r-v}\left(\begin{array}{l}
r \\
v
\end{array}\right) f(x+v h) .
\end{gathered}
$$

THEOREM 2. Let $f$ satisfy

$$
\int_{0}^{\delta} \frac{\omega_{r}(f, t)_{X} p}{t^{s+1}} d t<\infty,
$$

where $s$ is a positive integer with $0 \leqslant s<r$. Then there is $c_{1}>0$ depending on $p, \gamma$ and $r$ only such that

$$
E_{n}(f)_{X^{p}} \leqslant c_{1}(1 / n)^{s(\gamma-1) / \gamma} \int_{0}^{(1 / n)^{(\gamma-1) / \gamma}} \frac{\omega_{r}(f, t)_{X^{p}}}{t^{s+1}} d t .
$$

Proof. If $F_{(r)}$ is an $r$ th indefinite integral of $f$, then we have the following integral representation:

$$
\Delta_{h}^{r}\left(F_{(r)}, x\right)=h^{r} \int_{0}^{r} f(x+h t) \Pi_{r}(t) d t,
$$

where $\Pi_{r}$ is the Peano kernel for the $r$ th divided difference, with the properties

$$
\Pi_{r}(t) \geqslant 0, \quad \int_{0}^{r} \Pi_{r}(t) d t=1
$$

Put

$$
g_{\delta}(x)=\int_{0}^{r}\left[f(x)+(-1)^{r+1} \Delta_{\delta t / n d}^{r} f(x) \Pi_{r}(t) d t\right.
$$

Using the above integral representation, we find that $g_{\delta}$ has a locally integrable $r$ th order derivative, and we have the identity

$$
g_{\delta}(x)=\left(\frac{r}{\delta}\right)^{r} \sum_{v=1}^{r}(-1)^{v+1}\left(\begin{array}{l}
r \\
v
\end{array}\right) \frac{1}{v^{r}} \Delta_{\delta v / r}^{r}\left(F_{(r)}, x\right) .
$$

Differentiating this identity $r$ times and using the Minkowski inequality, we obtain

$$
\begin{gathered}
\delta^{r}\left\|g_{\delta}^{(r)}\right\|_{X} p \leqslant(2 r)^{r} \omega_{r}(f, \delta)_{X}, \\
\left\|f-g_{\delta}\right\|_{X \delta} \leqslant \omega_{r}(f, \delta)_{X} p .
\end{gathered}
$$


We now use $g_{\delta}$ as an intermediate approximation of $f$ in the following way:

$$
\begin{aligned}
E_{n}(f)_{X_{n}^{p}} & \leqslant E_{n}\left(f-g_{\delta}\right)_{X_{n}^{p}}+E_{n}\left(g_{\delta}\right)_{X_{n}^{p}} \\
& \leqslant\left\|f-g_{\delta}\right\|_{X_{n}^{p}}+c_{2}(1 / n)^{(\gamma-1) / \gamma}\left\|g_{\delta}^{(r)}\right\|_{X_{n}^{p}},
\end{aligned}
$$

where in the last inequality we have used Theorem 1 . Let $\delta=(1 / n)^{(\gamma-1) / \gamma}$. Then the above inequalities show that

$$
E_{n}(f)_{X_{n}^{p}} \leqslant c_{3} \omega_{r}\left(f,(1 / n)^{(\gamma-1) / \gamma}\right)_{X} p
$$

We now show how to extend the estimates of best approximations from the intervals $I_{n}$ to the whole real line.

For a given positive integer $n$, choose $m$ so that $2^{m} \leqslant n<2^{m+1}$ and find an algebraic polynomial $P_{n}$ of degree at most $n-1$ such that

$$
E_{n}(f)_{X{ }_{2 n}^{p}}=\left\|f-P_{n}\right\|_{X_{2 n}^{p}} .
$$

Recall that $X_{n}^{p}=L^{p}\left(I_{n}, \varrho\right), I_{n}=\left[-c n^{1 / \gamma}, c n^{1 / v}\right], c>0$. As shown in [10], there is $c>0$ such that for all algebraic polynomials $Q_{n}$ of degree at most $n-1$ we have

$$
\left\|Q_{n}\right\|_{X^{p}} \leqslant c_{4}\left\|Q_{n}\right\|_{X_{n}^{p}}
$$

where $c_{4}>0$ depends on $p$ and $\gamma$ only. Set $Q_{1}=P_{1}, Q_{2^{v}}=P_{2^{v}}-P_{2^{v-1}}$. Then

$$
\begin{aligned}
\left\|\sum_{v=m+1}^{\infty} Q_{2 v}\right\|_{X^{p}} & \leqslant c_{4} \sum_{\nu=m+1}^{\infty}\left\|Q_{2^{v}}\right\|_{X_{2^{v}}^{p}} \leqslant 2 c_{4} \sum_{\nu=m}^{\infty} E_{2^{v}}(f)_{X_{2^{v+1}}^{p}} \\
& \leqslant c_{5} \sum_{v=m}^{\infty} \omega_{r}\left(f, 2^{-(v+1)(\gamma-1) / \gamma}\right)_{X^{p}} \\
& \leqslant c_{6} 2^{-(m+1) s(\gamma-1) / \gamma} 2^{-(m+1)(\gamma-1) / \gamma} \cdot \int_{0} \frac{\omega_{r}(f, t)_{X^{p}}}{t^{s+1}} d t<\infty .
\end{aligned}
$$

Consequently, the series $\sum_{v=0}^{\infty} Q_{2^{v}}$ is convergent in the $X^{p}$ norm and

$$
E_{n}(f)_{X^{p}} \leqslant\left\|f-Q_{2^{m}}\right\|_{X^{p}} \leqslant c_{6}(1 / n)^{s(\gamma-1) / \gamma} \int_{0}^{(1 / n)^{(\gamma-1) / \gamma}} \frac{\omega_{r}(f, t)_{X^{p}}}{t^{s+1}} d t
$$

which completes the proof of Theorem 2.

THEOREM 3. If $f \in X^{p}$ satisfies

$$
E_{n}(f)_{X^{p}} \leqslant \omega\left((1 / n)^{(\gamma-1) / \gamma}\right), \quad n=1,2, \ldots,
$$

where $\omega$ is a function of the type of the r-th modulus of smoothness, then there is $c_{1}>0$ depending only on $p, \gamma, r$ and $\omega$ such that

$$
\omega_{r}(f, \delta)_{X^{p}} \leqslant \delta^{r} c_{1} \int_{\delta}^{1} \frac{\omega(t)}{t^{r+1}} d t .
$$


Proof. For a given $h>0$ we choose a positive integer $m$ such that $2^{-m(\gamma-1) / \gamma}<h \leqslant 2^{-(m-1)(\gamma-1) / \gamma}$ and find algebraic polynomials $P_{n}$ of degree at most $n-1$ satisfying

$$
E_{n}(f)_{X^{p}}=\left\|f-P_{n}\right\|_{X^{p}}
$$

Further, put $Q_{1}=P_{1}, Q_{2^{v}}=P_{2^{v}}-P_{2^{v-1}}$. Then

$$
\begin{aligned}
\left\|\Delta_{h}^{r}(f)\right\|_{X p^{p}} & \leqslant\left\|\Delta_{h}^{r}\left(f-P_{2^{m}}\right)\right\|_{X_{h}^{p}}+\left\|\Delta_{h}^{r}\left(P_{2^{m}}\right)\right\|_{X p_{h}^{p}} \\
& \leqslant 2^{r} E_{n}(f)_{X^{p}}+\sum_{v=1}^{m}\left\|\Delta_{h}^{r}\left(Q_{2^{v}}\right)\right\|_{X p_{h} .}
\end{aligned}
$$

Since

$$
\Delta_{h}^{r}\left(Q_{2^{v}}, x\right)=\int_{0}^{h} \ldots \int_{0}^{h} Q_{2^{v}}^{(r)}\left(x+t_{1}+\ldots+t_{r}\right) d t_{1} \ldots d t_{r},
$$

it follows by applying Freud's inequality [6] for the derivative of an algebraic polynomial:

$$
\left\|Q_{2^{v}}^{(r)}\right\|_{X^{p}} \leqslant c_{2} n^{r(\gamma-1) / \gamma}\left\|Q_{2} v\right\|_{X^{p}}
$$

that

$$
\left\|\Delta_{h}^{r}\left(Q_{2^{v}}\right)\right\|_{X}{ }_{h} \leqslant c_{3} h^{r} 2^{r v(\gamma-1) / \gamma}\left\|Q_{2^{v}}\right\|_{X^{p}} .
$$

Consequently, by the assumptions of the theorem,

$$
\begin{gathered}
\sum_{\nu=1}^{m}\left\|\Delta_{h}^{r}\left(Q_{2} v\right)\right\|_{X_{h}^{p}} \leqslant c_{4} h^{r} \sum_{\nu=0}^{m} 2^{r v(\gamma-1) / \gamma} \omega\left(2^{-v(\gamma-1) / \gamma}\right) \\
\leqslant c_{5} h^{r} \int_{2^{-m}(\gamma-1) / \gamma}^{1} \frac{\omega(t)}{t^{r+1}} d t, \\
E_{2^{m}}(f)_{X^{p}} \leqslant \omega\left(2^{-m(\gamma-1) / \gamma}\right) \leqslant c_{6} h^{r} \int_{2^{-m(\gamma-1) / \gamma}}^{1} \frac{\omega(t)}{t^{r+1}} d t .
\end{gathered}
$$

This finishes the proof of Theorem 3.

\section{References}

[1] M. M. Dzhrbashchyan, Some problems in the theory of weighted polynomial approximation in the complex domain, Mat. Sb. 36 (78) (3) (1955), 353-440 (in Russian).

[2] V. K. Dzyadyk, On constructive characterization of functions satisfying the condition $\operatorname{Lip}(0<\alpha<1)$ on a finite interval of the real line, Izv. Akad. Nauk SSSR Ser. Mat. 20 (2) (1956), 623-642 (in Russian).

[3] V. M. Fedorov, Constructive characterization of functions satisfying the Lipschitz condition on the half-line, Vestnik Moskov. Univ. Ser. I Mat. Mekh. 1983, no. 2, 64-69 (in Russian). 
[4] V. M. Fedorov, Some problems of approximation theory, Thesis, Moscow 1983 (in Russian).

[5] G. Freud, On polynomial approximation with respect to general weights, Lecture Notes in Math. 399, Springer, 1974, 149-179.

[6] -, On Markov-Bernstein type inequalities and their applications, J. Approx. Theory 19 (1) (1977), 22-37.

[7] -, Orthogonal Polynomials, Akademiai Kiadó, Budapest 1971.

[8] G. Fre u d, A. Giroux and Q. I. Rahman, Sur l'approximation polynomiale avec poids $\exp (-|x|)$, Canad. J. Math. 30 (2) (1978), 358-372.

[9] M. G. Krein and A. A. Nudel'man, Markov's Problem of Moments and Extremal Problems, Moscow 1973 (in Russian).

[10] G. P. Nevai, Polynomials orthogonal on the real line with weight $|x|^{\alpha} e^{-|x|^{\gamma}}$, Acta Math. Acad. Sci. Hungar. 24 (1973), 335-342 (in Russian).

[11] S. M. Nik ol'skii, On the best approximation of Lipschitz functions by polynomials, lzv. Akad. Nauk SSSR Ser. Mat. 10 (4) (1946), 295-318 (in Russian).

[12] M. K. Potapov, On the Jackson theorems in the $L_{p}$ norm, Dokl. Akad. Nauk SSSR (6) (1956), 1185-1188 (in Russian).

[13] - . Some prohlems of best approximation in the $L_{p}$ norm. Thesis, 1956 (in Russian).

[14] A. F. Timan, A strengthening of Jackson's theorem on best approximation of continuous functions by polynomials on a tinite interval of the real line, Dokl. Akad. Nauk SSSR 78 (1) (1951), 17-20 (in Russian).

Translated from the Russian by Jerzy Trzeciak

Presented to the Semester Approximation and Function Spaces

February 27-May 27, 1986 